\title{
Outcomes of patient self-referral for the diagnosis of several rare inherited kidney diseases
}

\author{
Anthony J. Bleyer, MD ${ }^{1,2,3}$, Kendrah Kidd, MS ${ }^{1,2}$, Victoria Robins, RN, BSN ${ }^{1}$, \\ Lauren Martin, MSW ${ }^{1}$, Abbigail Taylor, BS ${ }^{1}$, Annie Santi, MD ${ }^{4}$, Georgeanna Tsoumas, BS ${ }^{5}$, \\ Alese Hunt, MD ${ }^{6}$, Elizabeth Swain, BS ${ }^{7}$, Marwan Abbas, MD ${ }^{8}$, Ebun Akinbola, MD ${ }^{9}$, Sri Vidya, MD ${ }^{10}$, \\ Shahriar Moossavi, MD ${ }^{1}$, Anthony J. Bleyer Jr., MD', Martina Živná, PhD², Hana Hartmannová, PhD²', \\ Kateřina Hodaňová, PhD², Petr Vylełal, PhD ${ }^{2}$, Miroslav Votruba, BS², Maegan Harden, PhD $^{3}$, \\ Brendan Blumenstiel, $\mathrm{PhD}^{3}$, Anna Greka, MD, PhD ${ }^{3,11,12}$ and Stanislav Kmoch, PhD ${ }^{1,2,3}$
}

Purpose: To evaluate self-referral from the Internet for genetic diagnosis of several rare inherited kidney diseases.

Methods: Retrospective study from 1996 to 2017 analyzing data from an academic referral center specializing in autosomal dominant tubulointerstitial kidney disease (ADTKD). Individuals were referred by academic health-care providers (HCPs) nonacademic HCPs, or directly by patients/families.

Results: Over 21 years, there were 665 referrals, with 176 (27\%) directly from families, 269 (40\%) from academic HCPs, and 220 (33\%) from nonacademic HCPs. Forty-two (24\%) direct family referrals had positive genetic testing versus $73(27 \%)$ families from academic HCPs and 55 (25\%) from nonacademic HCPs $(P=0.72)$. Ninety-nine percent of direct family contacts were white and resided in zip code locations with a mean median income of $\$ 77,316 \pm 34,014$ versus US median income $\$ 49,445$.
Conclusion: Undiagnosed families with Internet access bypassed their physicians and established direct contact with an academic center specializing in inherited kidney disease to achieve a diagnosis. Twenty-five percent of all families diagnosed with ADTKD were the result of direct family referral and would otherwise have been undiagnosed. If patients suspect a rare disorder that is undiagnosed by their physicians, actively pursuing selfdiagnosis using the Internet can be successful. Centers interested in rare disorders should consider improving direct access to families.

Genetics in Medicine (2020) 22:142-149; https://doi.org/10.1038/s41436019-0617-8

Keywords: internet; rare disease; autosomal dominant tubulointerstitial kidney disease; uromodulin; mucin-1

\section{INTRODUCTION}

Poor access to health care often refers to individuals in need of care who cannot obtain it for socioeconomic reasons. Individuals with rare disorders also have difficulty accessing proper care, but their obstacles to care are unique. While it is estimated that $10 \%$ of individuals suffer from a rare disease, ${ }^{1}$ each disease is unique, preventing a common pathway to diagnosis. Often, the patient's primary physician is unaware of the correct diagnosis and the presentation of a rare disorder. Other factors preventing a physician's search for a diagnosis include time constraints from a high patient caseload, a simple lack of interest, or a belief that securing the diagnosis will not alter the patient's treatment plan. Specialists likewise may be unable to identify the disorder and do not explore further. If a researcher with expertise is identified, their practice may be very far from the patient, possibly on another continent. The plight of parents of children with undiagnosed, rare disorders has been described in the lay literature. ${ }^{2,3}$ Even physician-parents may be unsure how to pursue a diagnosis. ${ }^{4}$ While there are anecdotal reports of families finding a diagnosis when their physicians were unable, ${ }^{5}$ there have been no systematic investigations of individuals with rare disorders and their path to diagnosis.

The Internet has become increasingly important in the selfdiagnosis of health conditions, ${ }^{6,7}$ and focused information on rare disorders may lead to increasing diagnoses of these

\footnotetext{
${ }^{1}$ Section on Nephrology, Wake Forest School of Medicine, Winston-Salem, NC, USA; ${ }^{2}$ Research Unit for Rare Diseases, Department of Pediatrics and Adolescent Medicine, First Faculty of Medicine, Charles University, Prague, Czech Republic; ${ }^{3}$ Broad Institute of Harvard Medical School and Massachusetts Institute of Technology, Cambridge, MA, USA ${ }^{4}$ Department of Anesthesiology, University of Pennsylvania School of Medicine, Philadelphia, PA, USA; ${ }^{5}$ Campbell University School of Osteopathic Medicine, Lillington, NC, USA; ${ }^{6}$ Children's Health of Carolina Pediatrics, Lumberton, NC, USA; ${ }^{7}$ West Virginia University School of Medicine, Morgantown, WV, USA; ${ }^{8}$ Giza Renal Center, Giza, Egypt; ${ }^{9}$ East Carolina University, Brody School of Medicine, Greenville, NC, USA; ${ }^{10}$ Peace Health Southwest Medical Center, Vancouver, WA, USA; ${ }^{11}$ Division of Nephrology and Vascular Biology Research Center, Beth Israel Deaconess Medical Center, Boston, MA, USA; ${ }^{12}$ Brigham and Women's Hospital and Harvard Medical School, Boston, MA, USA. Correspondence: Anthony J. Bleyer (ableyer@wakehealth.edu)
} 
conditions. The Internet provides the patient with a rare disorder the opportunity to find the rare specialist interested in this disorder.

\section{Background}

Since 1999, our group has studied three rare genetic conditions that result in autosomal dominant tubulointerstitial kidney disease (ADTKD). ${ }^{8}$ The cardinal manifestations of these disorders include autosomal dominant inheritance of chronic kidney disease and a bland urinary sediment. Autosomal dominant inheritance results in many affected family members. Patients proceed to end-stage kidney disease requiring a kidney transplant or dialysis between the third and seventh decades. ADTKD-MUC1 (OMIM 174000) is due to pathogenic variants in the MUC1 gene and has no other associated symptoms. ${ }^{9}$ ADTKD-UMOD (OMIM 162000, 603860 ) is caused by pathogenic variants in the UMOD gene $^{10}$ encoding uromodulin and is associated with a high prevalence of gout. ADTKD-REN (OMIM 613092) is caused by pathogenic variants in the gene encoding renin ${ }^{11}$ and is associated with childhood anemia, gout, and hyperkalemia. These conditions present a diagnostic challenge because clinical findings are nonspecific, and the conditions are rare, recently identified, and not well known to nephrologists.

In 1999, there were fewer than ten US families with ADTKD described in the literature. After identification of the genetic cause of ADTKD-UMOD ${ }^{10}$ in 2002, a concerted attempt was made to identify as many ADTKD families as possible, communicating not only with health-care providers (HCPs), but also directly with families through Internet webpages. ${ }^{12}$ In 2009, pathogenic variants in $R E N^{11}$ and in 2014 pathogenic variants in $M U C 1^{9}$ were identified as causes of ADTKD. Due to complexities in sequencing MUC1, the Broad Institute is the only laboratory in the US that provides approved clinical testing for ADTKD-MUC1. ${ }^{13}$ All samples tested at the Broad Institute are first received and processed at Wake Forest School of Medicine. Thus, this investigation included all individuals in the US who underwent clinical genetic testing for ADTKD-MUC1.

The purpose of this analysis was to determine whether providing families with suspected rare disorders direct access to a research team through the Internet would result in genetic diagnoses of ADTKD and to compare direct access to families with indirect access to genetic testing through HCPs. As there was no diagnostic testing for these disorders prior to gene identification and we were the primary clinical academic center in in the United States interested in and actively trying to identify families with these disorders, we were also able to characterize the natural history of how rare conditions are diagnosed after they are definitively characterized.

\section{Recruitment}

\section{MATERIALS AND METHODS}

The following multifaceted approach was used to educate and generate potential referrals: publication of articles, ${ }^{14}$ reviews, ${ }^{15}$ and a Kidney Disease: Improving Global Outcomes (KDIGO) consensus report; ${ }^{16}$ presentations at national meetings and invited lectures at academic centers; and publication in other venues, including GeneReviews, Renal and Urology News', and the medical information resource UpToDate. In 2003, 1000 letters were mailed to US nephrologists requesting referral of any families with a suspected diagnosis of ADTKD. Several times over the last decade, Wake Forest School of Medicine sent brochures that included articles on ADTKD to US nephrologists. To encourage affected individuals or their families to contact us directly, a webpage was created. ${ }^{12}$ Information was also provided through the National Organization of Rare Disorders. ${ }^{17}$

\section{Family evaluation}

$U M O D$ and REN genetic analysis was performed by commercial laboratories or the laboratory of S.K. ${ }^{15}$ Genetic testing for ADTKD-MUC1 has previously been described. ${ }^{13}$

Figures 1, S1, S2, and S3 provide flow diagrams of the evaluation process. All referrals were evaluated by the lead author. The study did not include data on bulk shipment of samples specifically for $M U C 1$ genetic analysis from international centers specializing in renal genetics. For HCP referrals, the HCP would provide patient contact information if the patient was agreeable. In some cases, a clinical diagnosis other than ADTKD was made (either before or after negative genetic testing), and the patients were referred for genetic testing elsewhere. If agreeable, patients were consented and arrangements were made for a local laboratory to obtain a blood sample for genetic analysis at no cost to the patient. If patients did not provide consent or provide blood samples after several reminders by email and phone over one year, they were considered to have declined participation. In all cases, genetic testing was provided free to patients. The characteristics of the person initially referred by HCPs or the first clinically affected family member who agreed to participate are described. Median income according to zip code was obtained from 2010 US Census data. ${ }^{18}$

Study data were collected and managed using REDCap electronic data capture tools hosted at Wake Forest School of Medicine. ${ }^{19}$ REDCap is a secure, web-based, National Institutes of Health (NIH)-sponsored application that supports confidential data capture for research studies.

This study was approved by the institutional review boards of Wake Forest School of Medicine; the First Faculty of Medicine, Charles University (Prague, Czech Republic); and the Broad Institute of MIT and Harvard (Cambridge, MA) and adhered to the Declaration of Helsinki.

\section{Statistical analysis}

Statistical analysis was performed with SAS statistical programming (Cary, NC). Chi-squared testing was used to compare the three referral groups. For continuous variables, a general linear model was created, with type III sums of squares reported and the direct family referral group selected as the reference group. 


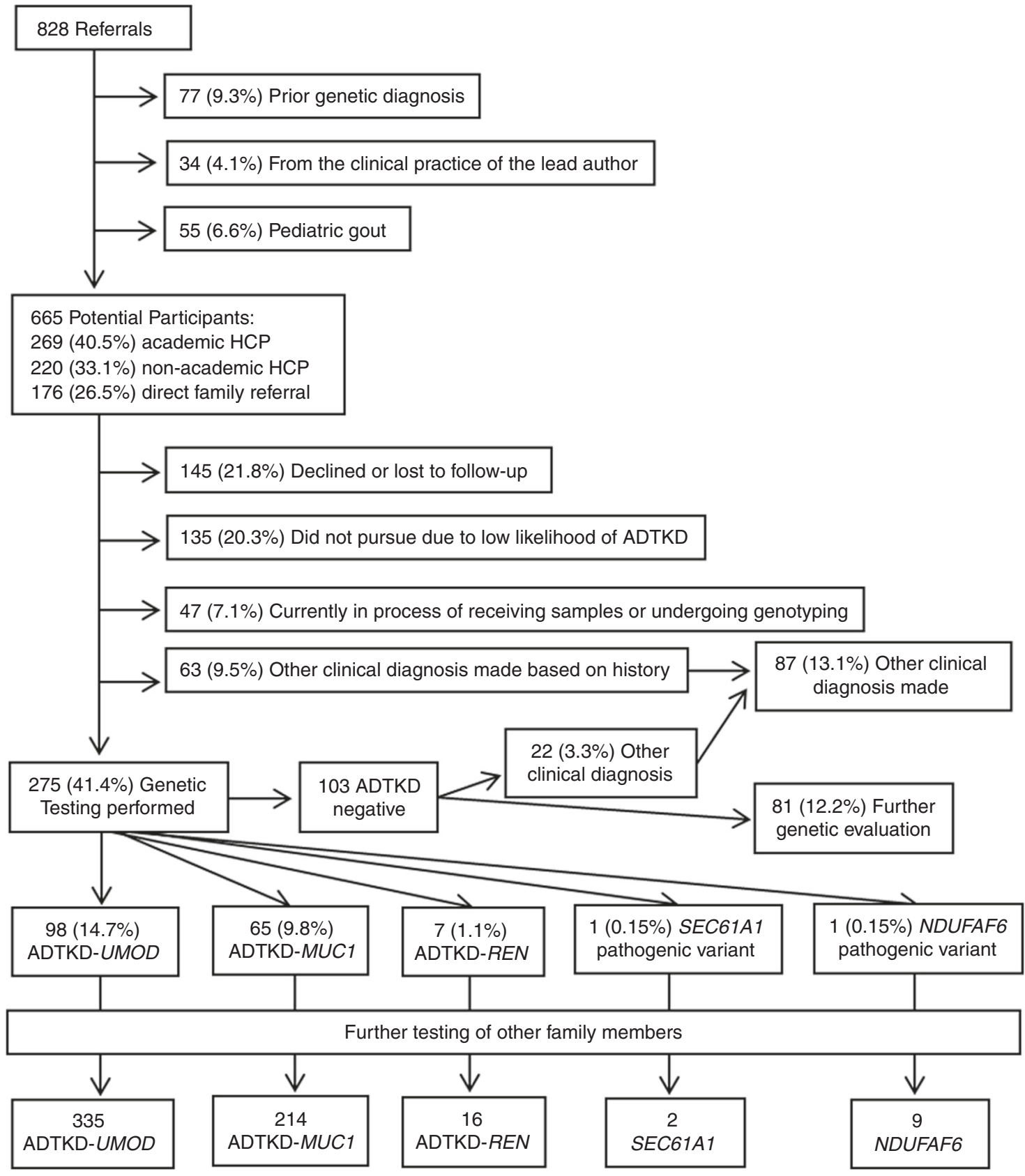

Fig. 1 Flow diagram of all referrals for evaluation for autosomal dominant tubulointerstitial kidney disease (ADTKD). Flow diagram of 828 family referrals for ADTKD evaluation. HCP health-care provider.

\section{RESULTS}

Over 21 years, there were 828 referrals. There were 77 individuals (9\%) who already had a genetic diagnosis, 34 individuals (4\%) referred directly from the first author's clinical practice, and $52(6.3 \%)$ referrals specifically for pediatric gout. These three groups were excluded from further analysis. The remaining 665 referrals included 269 (41\%) from HCPs at academic institutions, $220(33 \%)$ from HCPs at nonacademic institutions, and 176 (27\%) direct family referrals, defined as an affected or unaffected member of a family who does not have a known diagnosis of inherited disease contacting us through our website due to concerns that they might have ADTKD, without the guidance or assistance from their health-care provider. Eight (1\%) referrals from family members who were also physicians were included in the direct family referral group.

Of the 489 HCP referrals, 408 (83\%) were from nephrologists, 46 (9\%) non-nephrologist physicians, 30 (6\%) geneticists or genetics counselors, and 5 (1\%) HCPs not classified to other groups. There were $37 \mathrm{HCPs}$ who referred 2 families, and 22 HCPs who referred 3 or more families. Referral sources are listed in Table S1. Multiple avenues led to referrals. Despite increasing availability of electronic media, $252(52 \%)$ referrals were the result of some form of personal 
contact, of which 97 (20\%) were through personal acquaintance with the lead author, $96(20 \%)$ were at the recommendation of a colleague, and 59 (12\%) resulted from personal contact at meetings or lectures. There were 103 (21\%) referrals via the Internet, including UpToDate ${ }^{\circledR}$ and GeneReviews ${ }^{\circledR}$. There were $370(76 \%)$ referrals from the United States, with 26 (5\%) from Canada and 14 (3\%) from Australia.

Of the 176 direct family referrals, $116(66 \%)$ of the initial contacts were female, and 108 (61\%) were affected personally. Of unaffected individuals referring their family, 27 (24\%) were parents and $14(13 \%)$ were spouses, with 46 (41\%) unknown. There were 134 (76\%) referrals from the United States, 7 (4\%) from India, and 4 (2\%) from Canada, with 2 or fewer referrals from 19 countries. All direct family referrals were through our website, except for one family that learned of our research through the Facebook page of an affected individual.

A higher number of affected family members could be a catalyst for trying to establish a diagnosis, especially in the case of direct family referrals. Therefore, the number of known, clinically affected individuals in each family was analyzed according to referral group. The number of affected family members was similar between groups (see Table S2), with $22 \%$ of direct family referrals having greater than five affected family members versus $25 \%$ for nonacademic HCPs and $28 \%$ for academic HCPs $(P=0.17)$.

Table 1 shows outcomes versus referral type. Thirty-one percent of direct family referrals were not pursued due to low likelihood of ADTKD versus $18 \%$ for academic HCP and $15 \%$ for nonacademic HCP referrals $(P=0.0003)$. Twenty-seven percent of nonacademic HCP and $22 \%$ of academic HCP referrals declined participation versus $17 \%$ of direct family referrals $(P=0.04)$. Twenty-four percent of direct family referrals underwent genetic testing that resulted in a diagnosis of ADTKD-UMOD/MUC1/REN versus $27 \%$ of academic HCP and $25 \%$ of nonacademic HCP referrals $(P=0.72)$. Two referrals from nonacademic providers led to the identification of other new genetic causes of kidney disease. $^{20,21}$ Heterozygous loss-of-function SEC1A1 missense variants resulted in chronic tubulointerstitial kidney disease, congenital anemia, and pre- and postnatal growth retardation in one family and chronic kidney disease, anemia, and neutropenia in another family. ${ }^{20}$ NDUFAF6 missense variants are responsible for autosomal recessive Fanconi syndrome associated with chronic kidney disease and progressive pulmonary fibrosis. ${ }^{21}$ Further testing of other family members (see Table S3) from direct family referrals resulted in the identification of 81 individuals with ADTKD-UMOD, 32 individuals with ADTKD-MUC1, and 3 with ADTKD-REN. Direct family referral resulted in the diagnosis of 116 of 565 (21\%) individuals from all families identified with ADTKD. These families would have remained undiagnosed if family members had not independently sought a diagnosis on the Internet.

Characteristics of the initial contacts from direct family referrals who underwent sample collection (see Table 2) included a high proportion of white race, female gender, and higher median income by zip code. Of direct family referrals, $99 \%$ were white versus $93 \%$ from academic and $92 \%$ of nonacademic referrals $(P=0.045)$. Six $(5 \%)$ academic HCP referrals were African American families versus 0 for both nonacademic HCPs and direct family referrals. The median income by zip code was substantially higher for direct family referrals $(\$ 77,316 \pm 34,014$ versus $\$ 65,301 \pm 29,741$ academic $[P=0.04]$ and $\$ 63,934 \pm 24,403$ nonacademic $[P=0.03])$. Most individuals were referred with very advanced kidney disease, with $93 \%$ having an estimated glomerular filtration rate $<45 \mathrm{ml} / \mathrm{min} / 1.73 \mathrm{~m}^{2}$ or on dialysis/transplanted.

Figures 2 and 3, Table S4, and Figs. S4, S5, S6, and S7 display the temporal and geographic distribution of referrals. While genetic testing was available for one form of ADTKD as early as 2002, referrals have continued to increase over time. Both patient and academic referrals from Internet sources increased in approximately 2009. Despite increasing Internet

Table 1 Outcomes according to referral type

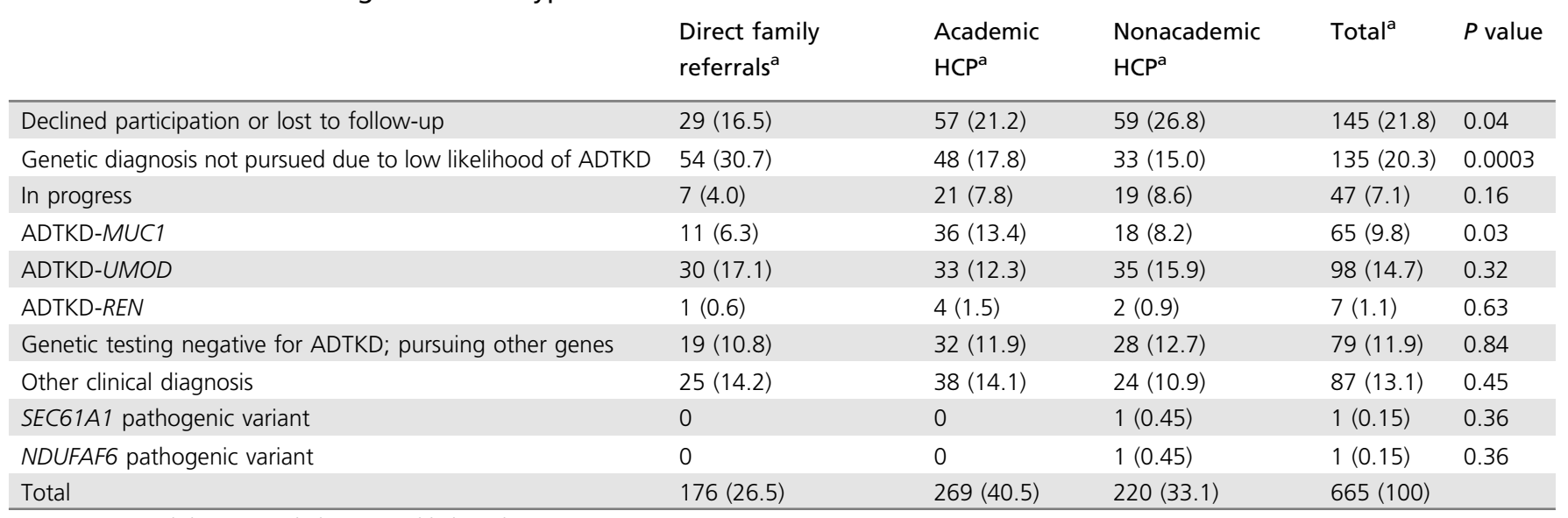

ADTKD autosomal dominant tubulointerstitial kidney disease.

${ }^{a}$ Data shown as number (\%). 
Table 2 Characteristics of first affected contact in families who underwent sample collection for the study

\begin{tabular}{|c|c|c|c|c|}
\hline & Direct family referrals & Academic HCP & Nonacademic HCP & $P$ value \\
\hline N & 68 & 120 & 101 & \\
\hline Gender ( $\%$ male) & $40(58.8)$ & $63(52.5)$ & $52(51.5)$ & 0.6 \\
\hline Age (years) & $47.9 \pm 15.8^{\mathrm{a}}$ & $43.7 \pm 17.2$ & $44.7 \pm 14.6$ & \\
\hline End-stage kidney disease at referral (\%) & $32(18.2)$ & $47(17.5)$ & $38(17.3)$ & 0.97 \\
\hline Estimated glomerular filtration rate $\left(\mathrm{ml} / \mathrm{min} / 1.73 \mathrm{~m}^{2}\right)^{\mathrm{b}}$ & $14.8 \pm 20.1^{c}$ & $22.4 \pm 27.1$ & $24.3 \pm 26.7$ & \\
\hline Mean median income by zip code $(\$)$ & $77,316 \pm 34,014^{d}$ & $65,301 \pm 29,741$ & $63,934 \pm 24,403$ & \\
\hline
\end{tabular}

HCP health-care provider.

aThere were no statistical differences between groups.

${ }^{b}$ Estimated glomerular filtration rate defined as $0 \mathrm{ml} / \mathrm{min} / 1.73 \mathrm{~m}^{2}$ for individuals with end-stage kidney disease at the time of referral.

'The mean estimated glomerular filtration rate was significantly different for direct family referrals versus nonacademic referrals $(P=0.03)$, but not significantly different versus academic referrals $(P=0.08)$

${ }^{\mathrm{d}}$ Median income was significantly different for direct family referrals versus nonacademic referrals $(P=0.03)$ and versus academic referrals $(P=0.04)$.

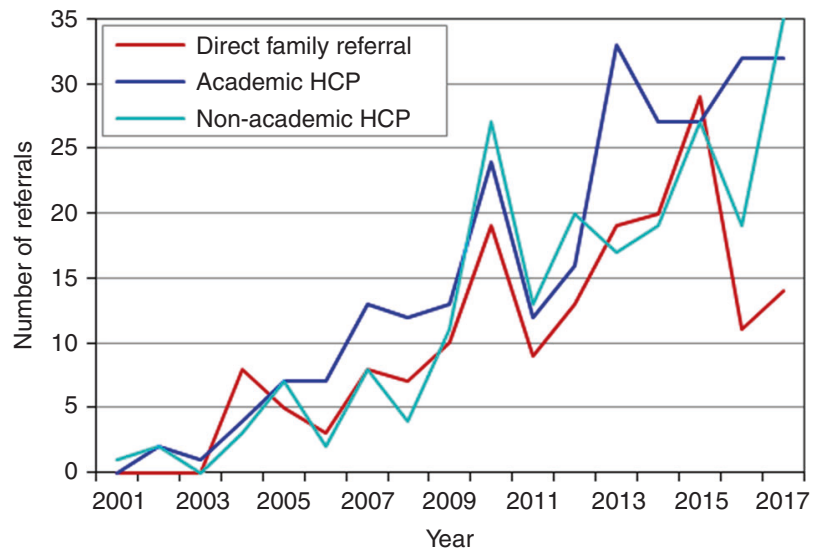

Fig. 2 Temporal distribution for referral type. Red represents direct family referrals, blue represents academic health-care providers (HCPs), and aqua represents nonacademic HCP.

resources, the primary source of HCP referrals continues to be some form of personal contact (personal knowledge of the author, referral at the suggestion of a colleague, or lectures), which has resulted in a rising number of referrals each year. Direct family referrals have decreased over the last two years, while HCP referrals have increased.

\section{DISCUSSION}

\section{Comparison with prior work}

While prior studies have questioned the effectiveness of Internet self-diagnosis, ${ }^{22,23}$ the current investigation showed that the Internet is an important tool for the self-diagnosis of rare disorders. Direct family referrals resulted in the diagnosis of 116 family members with ADTKD, none of whom would have received a diagnosis at that time if family members had not pursued self-diagnosis. Direct family referral via the Internet contributed $29 \%$ of the families and $21 \%$ of cases of these uncommon diseases, a major resource for clinical characterization and research. Importantly, patients originating from direct family referrals had similar frequencies of

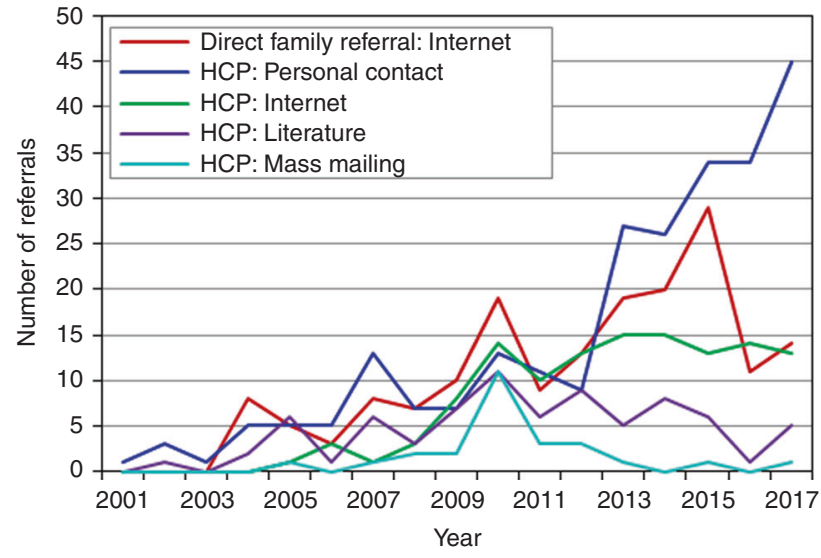

Fig. 3 Temporal distribution for method of referral. Red represents direct family referral via the Internet, with all family referrals being generated through Internet searches. Blue represents health-care provider (HCP) personal contact, including personal contact between the first author and the provider, colleague referral, and also lectures given by the first author. Green represents HCP referrals via Internet searches. Purple represents HCP referrals via reading of the literature. Aqua represents HCP referrals via mass mailing.

positive genetic diagnoses as referrals from HCPs. These observations provide insights about not only the selfdiagnosis of rare inherited kidney diseases, but also the increasing empowerment of patients and families with rare disorders. $^{24}$

\section{Principal results}

This article highlights the strengths and weaknesses of direct Internet referral of individuals with rare disorders. Advantages include low cost, low manpower requirements for initial referral, and the ability to bypass nondiagnosis by physicians. We observed that patients made judicious decisions about initiating contact. Based on clinical characteristics, some conditions may lend themselves better to self-diagnosis. Family members were more likely to diagnose ADTKD-UMOD than ADTKD-MUC1 (see Table 1). 
ADTKD-UMOD is more easily researched on the Internet due to the presence of both gout and inherited kidney disease, while ADTKD-MUC1 is only associated with inherited kidney disease, making Internet searches nonspecific and more difficult. The presence of many affected family members in autosomal disorders provides more exposure to physicians and/or family members who might be interested to pursue diagnosis. Thus, $36 \%$ of our referrals were from families with four or more affected individuals. Autosomal recessive disorders, with only one or two affected individuals in each family, may be more difficult to diagnose.

This article also characterized the natural history of diagnosis once the genetic causes of ADTKD were identified. Though genetic testing was available for the first of these disorders in 2002, referrals from HCPs continue to increase over a decade later. Direct family referrals have begun to decrease, possibly due to better recognition by HCPs. Despite the increasing availability of Internet resources, physician education about these rare disorders appears to occur primarily through personal interaction, resulting in increasing referrals over time.

\section{Limitations}

A shortcoming of our study is that we only studied one center that specialized in ADTKD, provided current knowledge on the topic, and provided a path to diagnosis. Patients searching for other conditions may be unable to find accurate information or a path to diagnosis. One report found that only $20 \%$ of English searches for health information yielded relevant results. ${ }^{25}$ Other studies have noted the poor quality of health information available on the Internet. ${ }^{22,23,26}$ Finding accurate information on the Internet is especially problematic for rare diseases, as approximately $50 \%$ of these disorders do not have a foundation providing specific patient information. ${ }^{1}$ Despite studies showing frequent use of the Internet by parents of children with rare conditions, ${ }^{27-32}$ one study reviewing 693 websites about rare diseases found that in general the quality of information provided was poor. ${ }^{33}$ Our results only describe outcomes that may occur when patients are provided with accurate information, personal contact, and a path to achieving diagnosis.

The potential use of the Internet for self-diagnosis also carries with it the inherent weaknesses of the digital divide. Almost all direct family referrals (99\%) providing genetic samples were from white families. The median income of the zip codes from the site of referrals was $\$ 77,316 \pm 34,014$, significantly higher than the 2010 US median household income of $\$ 49,445 .^{34}$ There were no direct family referrals from African Americans, and few individuals from non-English speaking countries. There is no evidence that the pathogenic variants causing ADTKD would have a difference in prevalence between races. Factors that affect access to health care through the Internet include availability of devices connected to the Internet. Individuals who use the Internet on a daily basis (e.g., for work) are likely to have better Internet search skills. In addition, our webpage information was provided only in English. An Italian study characterizing individuals who used the Internet to access a federation of associations of patients with rare diseases found that users were more likely to be female $(68 \%)$, have higher education, and use the Internet at work (74\%). Sixty-two percent of the respondents stated that the Internet helped them to achieve a diagnosis.

African Americans are more likely to have misgivings about research ${ }^{35}$ and therefore may have been less likely to contact us, even though kidney disease is much more prevalent in African Americans. In a focus group study of African Americans' views on the trustworthiness of physicians, Jacobs et al. reported the importance of interpersonal competence of physicians. ${ }^{36}$ Providing a more interpersonal experience on the Internet could include the use of more video material and providing faster methods of direct contact. Screening for genetic disorders in patients in dialysis centers would also provide a better interpersonal experience and enhance access to individuals across the digital divide.

African Americans were also less likely to be referred by their health-care providers. The high prevalence of chronic kidney disease in African Americans may have contributed to decreased consideration of a genetic diagnosis. Decreased access to health-care providers may have also contributed.

Despite many resources in the literature and on the Internet, $52 \%$ of HCP referrals were based on personal acquaintance with the lead author, lectures, or conversations with colleagues who had a personal knowledge of our research. Once nephrologists referred one family with ADTKD, they were likely to refer others. Unfortunately, most individuals from all three referral groups only undertook to establish a diagnosis when kidney disease was quite advanced and patients were near dialysis.

Strengths of this article include that this center was one of very few referral centers involved in the diagnosis of ATKD$U M O D$ and the only center arranging clinical genetic testing for ADTKD-MUC1. Weaknesses of this study include the unknown prevalence of this disorder. An Austrian study by Lhotta and colleagues ${ }^{37}$ estimated a prevalence of ADTKD$U M O D$ of 1.67 cases per million. Given a US population of 323 million, one would expect approximately 540 cases of ADTKD-UMOD, compared with the 283 cases that were identified. The relative contributions of our multifaceted interventions could not be determined. We could not account for how many families reviewed our website information and presented it to their physicians or how many individuals underwent commercial testing. We know that there were 2620 unique page views of our webpages in 2017. Similarly, for the National Organization of Rare Disorders (NORD) ADTKD website, there were 1518 unique page views in 2017 (Marsha Lanes, NORD, personal communication). Of note, 8.2 million users visited NORD webpages in 2017. We do not know how many families were tested at commercial laboratories for ADTKD-UMOD, though we know that all clinical diagnoses for ADTKD$M U C 1$ were only made in our laboratory. 


\section{Conclusions}

Despite these limitations, this investigation highlights the importance of providing direct knowledge and access to individuals with undiagnosed rare disorders through the Internet. Such direct access provided many families with a genetic diagnosis that had eluded them for generations. These families in turn provided a significant proportion of individuals willing to participate in clinical research. We believe that endeavors such as the Broad Institute Rare Genomes Project are likely to further empower patients to find the genetic causes of rare inherited diseases. ${ }^{38}$ For aid in the diagnosis of rare inherited kidney diseases, please contact ableyer@wakehealth.edu.

\section{SUPPLEMENTARY INFORMATION}

The online version of this article (https://doi.org/10.1038/s41436019-0617-8) contains supplementary material, which is available to authorized users.

\section{ACKNOWLEDGEMENTS}

This work was supported by $\mathrm{NIH}-\mathrm{National}$ Institute of Diabetes and Digestive and Kidney Diseases (NIDDK_ R21 DK106584), project NV17-29786A from the Ministry of Health of the Czech Republic, LQ1604 NPU II from the Ministry of Education of the Czech Republic, and by institutional programs of Charles University in Prague (UNCE 204064, PROGRES-Q26/LF1 and SVV 260367/2017), and the Carlos Slim Foundation.

\section{DISCLOSURE}

The authors declare no conflicts of interest.

Publisher's note: Springer Nature remains neutral with regard to jurisdictional claims in published maps and institutional affiliations.

\section{REFERENCES}

1. Global Genes. Rare diseases: facts and statistics. https://globalgenes.org/ rare-diseases-facts-statistics/. Accessed 22 July 2019.

2. Bigelow B. Tess is not alone: a USP7 story. 2017. Accessed 22 July 2019.

3. Mnookin S. One of a kind: what do you do if your child has a condition that is new to science? New Yorker. 14 July 2014. https://www. newyorker.com/magazine/2014/07/21/one-of-a-kind-2. Accessed 22 July 2019.

4. Rule ARL. I am that parent. JAMA. 2018;319:445.

5. Bouwman MG, Teunissen QG, Wijburg FA, Linthorst GE. 'Doctor Google' ending the diagnostic odyssey in lysosomal storage disorders: parents using internet search engines as an efficient diagnostic strategy in rare diseases. Arch Dis Child. 2010;95:642-644.

6. Patsos M. MSJAMA: the Internet and medicine: building a community for patients with rare diseases. JAMA. 2001;285:805.

7. Kuehn BM. More than one-third of US individuals use the Internet to selfdiagnose. JAMA. 2013;309:756-757.

8. Bleyer AJ, Kidd K, Zivna M, Kmoch S. Autosomal dominant tubulointerstitial kidney disease. Adv Chronic Kidney Dis. 2017; 24:86-93.

9. Kirby A, Gnirke A, Jaffe DB, et al. Mutations causing medullary cystic kidney disease type 1 lie in a large VNTR in MUC1 missed by massively parallel sequencing. Nat Genet. 2013:45:288-393.

10. Hart TC, Gorry MC, Hart PS, et al. Mutations of the UMOD gene are responsible for medullary cystic kidney disease 2 and familial juvenile hyperuricaemic nephropathy. J Med Genet. 2002;39:882-892.
11. Zivna M, Hulkova H, Marignon M, et al. Dominant renin gene mutations associated with early-onset hyperuricemia, anemia, and chronic kidney failure. Am J Human Genet. 2009;85:204-213.

12. Bleyer AJ: Uromodulin Kidney Disease. https://www.wakehealth.edu/ Condition/u/Uromodulin-Kidney-Disease. Accessed 22 July 2019.

13. Blumenstiel B, Defelice M, Birsoy O, et al. Development and validation of a mass spectrometry-based assay for the molecular diagnosis of mucin-1 kidney disease. J Mol Diagn. 2016;18:566-571.

14. Bleyer AJ, Kmoch S, Antignac C, et al. Variable clinical presentation of an MUC1 mutation causing medullary cystic kidney disease type 1. Clin J Am Soc Nephrol. 2014;9:527-535.

15. Bleyer AJ, Hart PS, Kmoch S. Hereditary interstitial kidney disease. Semin Nephrol. 2010;30:366-373.

16. Eckardt KU, Alper SL, Antignac C, et al. Autosomal dominant tubulointerstitial kidney disease: diagnosis, classification, and management-A KDIGO consensus report. Kidney Int. 2015;88: 676-683.

17. National Organization of Rare Disorders. Autosomal dominant tubulointerstitial kidney disease. 2017. https://rarediseases.org/rare-diseases/ autosomal-dominant-interstitial-kidney-disease/. Accessed 22 July 2019.

18. United States Census Bureau. American Fact Finder. https://factfinder. census.gov/faces/nav/isf/pages/index.xhtml. Accessed 22 July 2019.

19. Harris PA, Taylor R, Thielke $R$, et al. Research electronic data capture (REDCap)-a metadata-driven methodology and workflow process for providing translational research informatics support. J Biomed Inform. 2009; 42:377-381.

20. Bolar NA, Golzio C, Zivna M, et al. Heterozygous loss-of-function SEC61A1 mutations cause autosomal-dominant tubulo-interstitial and glomerulocystic kidney disease with anemia. Am J Hum Genet. 2016; 99:174-187.

21. Hartmannova $\mathrm{H}$, Piherova $L$, Tauchmannova $\mathrm{K}$, et al. Acadian variant of Fanconi syndrome is caused by mitochondrial respiratory chain complex I deficiency due to a non-coding mutation in complex I assembly factor NDUFAF6. Hum Mol Genet. 2016;25:4062-4079.

22. Semigran $\mathrm{HL}$, Linder JA, Gidengil C, Mehrotra A. Evaluation of symptom checkers for self diagnosis and triage: audit study. BMJ. 2015;351:h3480.

23. Bisson LJ, Komm JT, Bernas GA, et al. How accurate are patients at diagnosing the cause of their knee pain with the help of a web-based symptom checker? Orthop J Sports Med. 2016;4: 2325967116630286

24. Ayme S, Kole A, Groft S. Empowerment of patients: lessons from the rare diseases community. Lancet. 2008;371:2048-2051.

25. Berland GK, Elliott MN, Morales LS, et al. Health information on the Internet: accessibility, quality, and readability in English and Spanish. JAMA. 2001;285:2612-2621.

26. Eysenbach G, Powell J, Kuss O, Sa ER. Empirical studies assessing the quality of health information for consumers on the world wide web: a systematic review. JAMA. 2002;287:2691-2700.

27. Nicholl H, Tracey $C$, Begley $T$, et al. Internet use by parents of children with rare conditions: findings from a study on parents' web information needs. J Med Internet Res. 2017;19:e51.

28. Morgan T, Schmidt J, Haakonsen C, et al. Using the internet to seek information about genetic and rare diseases: a case study comparing data from 2006 and 2011. JMIR Res Protoc. 2014;3:e10.

29. Hamilton JG, Hutson SP, Frohnmayer AE, et al. Genetic informationseeking behaviors and knowledge among family members and patients with inherited bone marrow failure syndromes. J Genet Couns. 2015;24:760-770

30. Schumacher KR, Stringer KA, Donohue JE, et al. Social media methods for studying rare diseases. Pediatrics. 2014;133:e1345-e1353.

31. Davies W. Insights into rare diseases from social media surveys. Orphanet J Rare Dis. 2016;11:151.

32. Babac A, Litzkendorf S, Schmidt $K$, et al. Shaping an effective health information website on rare diseases using a group decision-making tool: inclusion of the perspectives of patients, their family members, and physicians. Interact J Med Res. 2017;6:e23.

33. Pauer F, Gobel J, Storf $\mathrm{H}$, et al. Adopting quality criteria for websites providing medical information about rare diseases. Interact J Med Res. 2016;5:e24.

34. United States Census Bureau. Income, poverty and health insurance coverage in the United States: 2010. September 2011. https://uww.census. gov/newsroom/releases/pdf/2010_Report.pdf. Accessed 22 July 2019. 
35. Scharff DP, Mathews KJ, Jackson $\mathrm{P}$, et al. More than Tuskegee: understanding mistrust about research participation. J Health Care Poor Underserved. 2010;21:879-897.

36. Jacobs EA, Rolle I, Ferrans CE, et al. Understanding African Americans' views of the trustworthiness of physicians. J Gen Intern Med. 2006;21:642-647.
37. Lhotta K, Piret SE, Kramar R, et al. Epidemiology of uromodulinassociated kidney disease-results from a nation-wide survey. Nephron Extra. 2012;2:147-158.

38. Broad Institute. Rare Genomes Project. https://raregenomes.org/home. Accessed July 172019. 